\title{
Approximating Iterations for Nonexpansive and Maximal Monotone Operators
}

\author{
Zhangsong Yao, ${ }^{1}$ Sun Young Cho, ${ }^{2}$ Shin Min Kang, ${ }^{3}$ and Li-Jun $\mathrm{Zhu}^{4}$ \\ ${ }^{1}$ School of Mathematics \& Information Technology, Nanjing Xiaozhuang University, Nanjing 211171, China \\ ${ }^{2}$ Department of Mathematics, Gyeongsang National University, Jinju 660-701, Republic of Korea \\ ${ }^{3}$ Department of Mathematics and RINS, Gyeongsang National University, Jinju 660-701, Republic of Korea \\ ${ }^{4}$ School of Mathematics and Information Science, Beifang University of Nationalities, Yinchuan 750021, China \\ Correspondence should be addressed to Sun Young Cho; ooly61@yahoo.co.kr and Shin Min Kang; smkang@gnu.ac.kr
}

Received 29 May 2014; Accepted 3 August 2014

Academic Editor: Poom Kumam

Copyright (C) 2015 Zhangsong Yao et al. This is an open access article distributed under the Creative Commons Attribution License, which permits unrestricted use, distribution, and reproduction in any medium, provided the original work is properly cited.

We present two algorithms for finding a zero of the sum of two monotone operators and a fixed point of a nonexpansive operator in Hilbert spaces. We show that these two algorithms converge strongly to the minimum norm common element of the zero of the sum of two monotone operators and the fixed point of a nonexpansive operator.

\section{Introduction}

Throughout, we assume that $\mathscr{H}$ is a real Hilbert space with inner product $\langle\cdot, \cdot\rangle$ and norm $\|\cdot\|$, respectively. Let $\mathscr{C} \subset \mathscr{H}$ be a nonempty closed convex set.

Definition 1. An operator $\mathbb{S}: \mathscr{C} \rightarrow \mathscr{C}$ is said to be nonexpansive if

$$
\|\mathbb{S} u-\mathbb{S} v\| \leq\|u-v\|
$$

for all $u, v \in \mathscr{C}$.

We denote by $\operatorname{Fix}(\mathbb{S})$ the set of fixed points of $\mathbb{S}$.

Definition 2. An operator $\mathbb{A}: \mathscr{C} \rightarrow \mathscr{H}$ is said to be $\xi$-inverse strong monotone if

$$
\langle A u-A v, u-v\rangle \geq \xi\|\mathbb{A} u-A v\|^{2}
$$

for some $\xi>0$ and for all $u, v \in \mathscr{C}$.

It is known that if $\mathbb{A}$ is $\xi$-inverse strong monotone, then $\mathbb{A}$ is $1 / \xi$-lipschitz, that is,

$$
\|A u-A v\| \leq \frac{1}{\xi}\|u-v\|,
$$

for all $u, v \in \mathscr{C}$. Furthermore,

$$
\begin{aligned}
& \|(I-\delta \mathbb{A}) u-(I-\delta \AA) v\|^{2} \\
& \quad \leq\|u-v\|^{2}+\delta(\delta-2 \xi)\|\mathbb{A} u-\mathbb{A} v\|^{2}, \quad \forall u, v \in \mathscr{C} .
\end{aligned}
$$

In particular, if $\delta \in(0,2 \xi)$, then $I-\delta \mathbb{A}$ is nonexpansive.

Let $\mathbb{B}: \mathscr{H} \rightarrow 2^{\mathscr{H}}$ be a set-valued operator. The effective domain of $\mathbb{B}$ is denoted by $\operatorname{dom}(\mathbb{B})$, that is, $\operatorname{dom}(\mathbb{B})=\{x \in$ $\mathscr{H}: \mathbb{B} x \neq \emptyset\}$.

Definition 3. A multivalued operator $\mathbb{B}$ is said to be a monotone on $\mathscr{H}$ if and only if

$$
\langle x-y, u-v\rangle \geq 0
$$

for all $x, y \in \operatorname{dom}(\mathbb{B}), u \in \mathbb{B} x$, and $v \in \mathbb{B} y$.

A monotone operator $\mathbb{B}$ on $\mathscr{H}$ is said to be maximal if and only if its graph is not strictly contained in the graph of any other monotone operator on $\mathscr{H}$. We denote by $\mathbb{B}^{-1} 0$ the set of the zero points of $\mathbb{B}$, that is, $\mathbb{B}^{-1} 0=\{x \in \mathscr{H}: 0 \in \mathbb{B} x\}$.

For $\lambda>0$, we define a single-valued operator

$$
J_{\lambda}^{\mathbb{B}}=(I+\lambda \mathbb{B})^{-1}: \mathscr{H} \longrightarrow \operatorname{dom}(\mathbb{B}),
$$


which is called the resolvent of $\mathbb{B}$ for $\lambda$. It is known that the resolvent $J_{\lambda}^{\mathbb{B}}$ is firmly nonexpansive, that is,

$$
\left\|J_{\lambda}^{\mathbb{B}} u-J_{\lambda}^{\mathbb{B}} v\right\|^{2} \leq\left\langle J_{\lambda}^{\mathbb{B}} u-J_{\lambda}^{\mathbb{B}} v, u-v\right\rangle,
$$

for all $u, v \in \mathscr{C}$ and $\mathbb{B}^{-1} 0=\operatorname{Fix}\left(J_{\lambda}^{\mathbb{B}}\right)$ for all $\lambda>0$.

In the present paper, we consider the variational inclusion of finding a zero $x \in \mathscr{H}$ of the sum of two monotone operators $\mathbb{A}$ and $\mathbb{B}$ such that

$$
0 \in \mathbb{A}(x)+\mathbb{B}(x),
$$

where $\mathbb{A}: \mathscr{H} \rightarrow \mathscr{H}$ is a single-valued operator and $\mathbb{B}: \mathscr{H} \rightarrow$ $2^{\mathscr{H}}$ is a set-valued operator. The set of solutions of problem (8) is denoted by $(\mathbb{A}+\mathbb{B})^{-1}(0)$.

Special Cases. (i) If $\mathscr{H}=\mathbb{R}^{m}$, then problem (8) becomes the generalized equation introduced by Robinson [1].

(ii) If $\mathbb{A}=0$, then problem ( 8 ) becomes the inclusion problem introduced by Rockafellar [2].

It is known that (8) provides a convenient framework for the unified study of optimal solutions in many optimization related areas including mathematical programming, complementarity, variational inequalities, optimal control, mathematical economics, equilibria, and game theory. Also various types of variational inclusions problems have been extended and generalized. For related work, please see [3-20].

Zhang et al. [21] introduced the following iterative algorithm for finding a common element of the set of solutions to the problem (8) and the set of fixed points of a nonexpansive operator:

$$
x_{n+1}=\alpha_{n} x_{0}+\left(1-\alpha_{n}\right) \mathbb{S} J_{\lambda}^{\mathbb{B}}\left(x_{n}-\lambda \mathbb{A} x_{n}\right),
$$

where $\mathbb{S}: \mathscr{C} \rightarrow \mathscr{C}$ is a nonexpansive operator. Under some mild conditions, they prove that the sequence $\left\{x_{n}\right\}$ converges strongly to $x^{*} \in \operatorname{Fix}(\mathbb{S}) \cap(\mathbb{A}+\mathbb{B})^{-1}(0)$.

Recently, Takahashi et al. [22] introduced another iterative algorithm for finding a zero of the sum of two monotone operators and a fixed point of a nonexpansive operator

$$
x_{n+1}=\beta_{n} x_{n}+\left(1-\beta_{n}\right) \mathbb{S}\left(\alpha_{n} x_{0}+\left(1-\alpha_{n}\right) J_{\lambda_{n}}^{\mathbb{B}}\left(x_{n}-\lambda_{n} \mathbb{A} x_{n}\right)\right)
$$

for all $n \geq 0$. Under some assumptions, they proved that the sequence $\left\{x_{n}\right\}$ converges strongly to a point of $\operatorname{Fix}(\mathbb{S}) \cap(\mathbb{A}+$ $\mathbb{B})^{-1}(0)$.

Motivated and inspired by (9) and (10), in the present paper, we suggest two algorithms

$$
\begin{array}{r}
x_{t}=J_{\lambda}^{\mathbb{B}}\left((1-t) \mathbb{S} x_{t}-\lambda \mathbb{A} \mathbb{S} x_{t}\right), \quad t \in(0,1), \\
x_{n+1}=\beta_{n} x_{n}+\left(1-\beta_{n}\right) J_{\lambda_{n}}^{\mathbb{B}}\left(\left(1-\alpha_{n}\right) \mathbb{S} x_{n}-\lambda_{n} \mathbb{A} \mathbb{S} x_{n}\right), \\
n \geq 0 .
\end{array}
$$

It is obvious that (12) is very different from (9) and (10). Furthermore, we prove that both (11) and (12) converge strongly to the minimum norm element in $\operatorname{Fix}(\mathbb{S}) \cap(\mathbb{A}+$ $\mathbb{B})^{-1} 0$. It should be pointed out that we do not use the metric projection in (11) and (12).

\section{Lemmas}

In this section, we collect several useful lemmas for our next section.

First, the following resolvent equality is well known.

Lemma 4. For $\lambda>0$ and $\lambda^{\dagger}>0$, one has

$$
J_{\lambda}^{\mathbb{B}} u=J_{\lambda^{\dagger}}^{\mathbb{B}}\left(\frac{\lambda^{\dagger}}{\lambda} u+\left(1-\frac{\lambda^{\dagger}}{\lambda}\right) J_{\lambda}^{\mathbb{B}} u\right), \quad \forall u \in \mathscr{H} .
$$

Lemma 5 (see [23]). Let $\mathscr{C} \subset \mathscr{H}$ be a closed convex set. Let $\mathbb{S}: \mathscr{C} \rightarrow \mathscr{C}$ be a nonexpansive operator. Then $\operatorname{Fix}(\mathbb{S})$ is a closed convex subset of $\mathscr{C}$ and the operator $I-\mathbb{S}$ is demiclosed at 0 .

Lemma 6 (see [24]). Let $\mathscr{X}$ be a Banach space. Let $\left\{u_{n}\right\} \subset \mathscr{X}$ and $\left\{v_{n}\right\} \subset \mathscr{X}$ be two bounded sequences. Let the sequence $\left\{\zeta_{n}\right\} \subset(0,1)$ satisfy $0<\underline{\lim }_{n \rightarrow \infty} \zeta_{n} \leq \overline{\lim }_{n \rightarrow \infty} \zeta_{n}<1$. Suppose $u_{n+1}=\left(1-\zeta_{n}\right) v_{n}+\zeta_{n} u_{n}$ for all $n \geq 0$ and $\overline{\lim }_{n \rightarrow \infty}\left(\| v_{n+1}-\right.$ $\left.v_{n}\|-\| u_{n+1}-u_{n} \|\right) \leq 0$. Then $\lim _{n \rightarrow \infty}\left\|u_{n}-v_{n}\right\|=0$.

Lemma 7 (see [25]). Let $\left\{\sigma_{n}\right\} \subset[0, \infty),\left\{\gamma_{n}\right\} \subset(0,1)$, and $\left\{\delta_{n}\right\} \subset \mathbb{R}$ be three sequences satisfying

$$
\sigma_{n+1} \leq\left(1-\gamma_{n}\right) \sigma_{n}+\delta_{n} \gamma_{n}
$$

If $\sum_{n=1}^{\infty} \gamma_{n}=\infty$ and $\overline{\lim }_{n \rightarrow \infty} \delta_{n} \leq 0$ (or $\sum_{n=1}^{\infty}\left|\delta_{n} \gamma_{n}\right|<\infty$ ), then $\lim _{n \rightarrow \infty} \sigma_{n}=0$.

\section{Strong Convergence Results}

Let $\mathscr{C} \subset \mathscr{H}$ be a nonempty closed convex set. Let $\mathbb{A}$ : $\mathscr{C} \rightarrow \mathscr{H}$ be a $\varrho$-inverse strong monotone operator. Let $\mathbb{B}$ be a maximal monotone operator on $\mathscr{H}$ such that $\operatorname{dom}(\mathbb{B}) \subset \mathscr{C}$. Let $\mathbb{S}: \mathscr{C} \rightarrow \mathscr{C}$ be a nonexpansive operator.

Pick up a constant $\tau \in(0,2 \varrho)$. For any $t \in(0,(2 \varrho-\tau) / 2 \varrho)$, we define an operator

$$
\psi(x)=J_{\tau}^{\mathbb{B}}((1-t) \mathbb{S}-\tau \mathbb{A} \mathbb{S}) x,
$$

for all $x \in \mathscr{C}$.

Since $J_{\tau}^{\mathbb{B}}, \mathbb{S}$, and $I-\tau \mathbb{A} /(1-t)$ are nonexpansive, we have

$$
\begin{aligned}
\|\psi(x)-\psi(y)\|= & \| J_{\tau}^{\mathbb{B}}\left((1-t)\left(I-\frac{\tau}{1-t} \mathbb{A}\right) \mathbb{S} x\right) \\
& -J_{\tau}^{\mathbb{B}}\left((1-t)\left(I-\frac{\tau}{1-t} \mathbb{A}\right) \mathbb{S} y\right) \| \\
\leq & (1-t) \|\left(I-\frac{\tau}{1-t} \mathbb{A}\right) \mathbb{S} x \\
& \quad-\left(I-\frac{\tau}{1-t} \mathbb{A}\right) \mathbb{S} y \| \\
\leq & (1-t)\|x-y\|,
\end{aligned}
$$

for any $x, y \in \mathscr{C}$. Hence $\psi$ is a contraction on $\mathscr{C}$. We use $x_{t}$ to denote the unique fixed point of $\psi$ in $\mathscr{C}$. Thus, $\left\{x_{t}\right\}$ satisfies the fixed point equation

$$
x_{t}=J_{\tau}^{\mathbb{B}}\left((1-t) \mathbb{S} x_{t}-\tau \mathbb{A} \mathbb{S} x_{t}\right) .
$$

Next, we give the convergence analysis of (17). 
Theorem 8. Assume that $\operatorname{Fix}(\mathbb{S}) \cap(\mathbb{A}+\mathbb{B})^{-1} 0 \neq \emptyset$. Then $\left\{x_{t}\right\}$ defined by (17) converges strongly, as $t \rightarrow 0+$, to the minimum norm element in $\operatorname{Fix}(\mathbb{S}) \cap(\mathbb{A}+\mathbb{B})^{-1}(0)$.

Proof. Choose any $z \in \operatorname{Fix}(\mathbb{S}) \cap(\mathbb{A}+\mathbb{B})^{-1}(0)$. It is obvious that $z=\mathbb{S} z=J_{\tau}^{\mathbb{B}}(z-\tau \mathbb{A} z)$ for all $\tau>0$. So, we have

$$
z=\mathbb{S} z=J_{\tau}^{\mathbb{B}}(z-\tau \mathbb{A} z)=J_{\tau}^{\mathbb{B}}\left(t z+(1-t)\left(I-\frac{\tau}{1-t} \mathbb{A}\right) \mathbb{S} z\right)
$$

for all $t \in(0,1)$.

From (17), we have

$$
\begin{aligned}
\left\|x_{t}-z\right\|= & \left\|J_{\tau}^{\mathbb{B}}\left((1-t)\left(I-\frac{\tau}{1-t} \mathbb{A}\right) \mathbb{S} x_{t}\right)-z\right\| \\
= & \| J_{\tau}^{\mathbb{B}}\left((1-t)\left(\mathbb{S} x_{t}-\frac{\tau}{1-t} \mathbb{A} \mathbb{S} x_{t}\right)\right) \\
& \quad-J_{\tau}^{\mathbb{B}}\left(t z+(1-t)\left(\mathbb{S} z-\frac{\tau}{1-t} \mathbb{A} \mathbb{S} z\right)\right) \| \\
\leq & \|(1-t)\left(\mathbb{S} x_{t}-\frac{\tau}{1-t} \mathbb{A} \mathbb{S} x_{t}\right) \\
& \quad-\left(t z+(1-t)\left(\mathbb{S} z-\frac{\tau}{1-t} \mathbb{A} \mathbb{S} z\right)\right) \| \\
= & \|(1-t)\left(\left(\mathbb{S} x_{t}-\frac{\tau}{1-t} \mathbb{A} \mathbb{S} x_{t}\right)\right. \\
\leq & \quad(1-t)\left\|\left(I-\frac{\tau}{1-t} \mathbb{A}\right) \mathbb{S} x_{t}-\left(I-\frac{\tau}{1-t} \mathbb{A}\right) \mathbb{S} z\right\| \\
& +t\|z\| \quad(1-t)\left\|x_{t}-z\right\|+t\|z\| .
\end{aligned}
$$

Hence, we get

$$
\left\|x_{t}-z\right\| \leq\|z\| .
$$

Thus, $\left\{x_{t}\right\}$ is bounded.

By (4) and (19), we derive

$$
\begin{aligned}
\left\|x_{t}-z\right\|^{2} \leq \|(1-t)( & \left(\mathbb{S} x_{t}-\frac{\tau}{1-t} \mathbb{A} \mathbb{S} x_{t}\right) \\
& \left.-\left(\mathbb{S} z-\frac{\tau}{1-t} \mathbb{A} \mathbb{S} z\right)\right)+t(-z) \|^{2} \\
\leq & (1-t) \|\left(\mathbb{S} x_{t}-\frac{\tau}{1-t} \mathbb{A} \mathbb{S} x_{t}\right) \\
& -\left(\mathbb{S} z-\frac{\tau}{1-t} \mathbb{A} \mathbb{S} z\right)\left\|^{2}+t\right\| z \|^{2} \\
= & (1-t)\left\|\left(\mathbb{S} x_{t}-\mathbb{S} z\right)-\frac{\tau}{1-t}\left(\mathbb{A} \mathbb{S} x_{t}-\mathbb{A} \mathbb{S} z\right)\right\|^{2} \\
& +t\|z\|^{2}
\end{aligned}
$$

$$
\begin{aligned}
& =(1-t)\left(\left\|\mathbb{S} x_{t}-\mathbb{S} z\right\|^{2}-\frac{2 \tau}{1-t}\right. \\
& \times\left\langle\mathbb{A} \mathbb{S} x_{t}-\mathbb{A} \mathbb{S} z, \mathbb{S} x_{t}-\mathbb{S} z\right\rangle \\
& \left.+\frac{\tau^{2}}{(1-t)^{2}}\left\|\mathbb{A} S x_{t}-\mathbb{A} \mathbb{S} z\right\|^{2}\right)+t\|z\|^{2} \\
& \leq(1-t)\left(\left\|\mathbb{S} x_{t}-\mathbb{S} z\right\|^{2}-\frac{2 \varrho \tau}{1-t}\left\|\mathbb{A} \mathbb{S} x_{t}-\mathbb{A} \mathbb{S} z\right\|^{2}\right. \\
& \left.+\frac{\tau^{2}}{(1-t)^{2}}\left\|\mathbb{A} \mathbb{S} x_{t}-\mathbb{A} \mathbb{S} z\right\|^{2}\right)+t\|z\|^{2} \\
& =(1-t)\left(\left\|\mathbb{S} x_{t}-\mathbb{S} z\right\|^{2}+\frac{\tau}{(1-t)^{2}}(\tau-2(1-t) \varrho)\right. \\
& \left.\times\left\|\mathbb{A} S x_{t}-\mathbb{A} S z\right\|^{2}\right)+t\|z\|^{2} \\
& \leq(1-t)\left\|x_{t}-z\right\|^{2}+\frac{\tau}{1-t}(\tau-2(1-t) \varrho) \\
& \times\left\|\mathbb{A} S x_{t}-\mathbb{A} \mathbb{S} z\right\|^{2}+t\|z\|^{2} .
\end{aligned}
$$

So,

$$
\begin{aligned}
& \frac{\tau}{1-t}(2(1-t) \varrho-\tau)\left\|\mathbb{A} \mathbb{S} x_{t}-\mathbb{A} z\right\|^{2} \\
& \quad \leq t\|z\|^{2}-t\left\|x_{t}-z\right\|^{2} \longrightarrow 0 .
\end{aligned}
$$

Since $2(1-t) \varrho-\tau>0$ for all $t \in(0,1-\tau / 2 \varrho)$, we obtain

$$
\lim _{t \rightarrow 0+}\left\|\mathbb{A} \mathbb{S} x_{t}-\mathbb{A} z\right\|=0 \text {. }
$$

Using the firm nonexpansivity of $J_{\tau}^{\mathbb{B}}$, we have

$$
\begin{aligned}
\left\|x_{t}-z\right\|^{2}= & \left\|J_{\tau}^{\mathbb{B}}\left((1-t) \mathbb{S} x_{t}-\tau \mathbb{A} \mathbb{S} x_{t}\right)-z\right\|^{2} \\
= & \left\|J_{\tau}^{\mathbb{B}}\left((1-t) \mathbb{S} x_{t}-\tau \mathbb{A} \mathbb{S} x_{t}\right)-J_{\tau}^{\mathbb{B}}(z-\tau \mathbb{A} z)\right\|^{2} \\
\leq & \left\langle(1-t) \mathbb{S} x_{t}-\tau \mathbb{A} \mathbb{S} x_{t}-(z-\tau \mathbb{A} z), x_{t}-z\right\rangle \\
= & \frac{1}{2}\left(\left\|(1-t) \mathbb{S} x_{t}-\tau \mathbb{A} \mathbb{S} x_{t}-(z-\tau \mathbb{A} z)\right\|^{2}\right. \\
& \quad+\left\|x_{t}-z\right\|^{2} \\
& \left.\quad-\left\|(1-t) \mathbb{S} x_{t}-\tau\left(\mathbb{A} \mathbb{S} x_{t}-\tau \mathbb{A} z\right)-x_{t}\right\|^{2}\right) .
\end{aligned}
$$

Note that

$$
\begin{aligned}
\left\|(1-t) \mathbb{S} x_{t}-\tau \mathbb{A} \mathbb{S} x_{t}-(z-\tau \mathbb{A} z)\right\|^{2} \\
=\|(1-t)\left(\left(\mathbb{S} x_{t}-\frac{\tau}{1-t} \mathbb{A} \mathbb{S} x_{t}\right)\right. \\
\left.-\left(\mathbb{S} z-\frac{\tau}{1-t} \mathbb{A} \mathbb{S} z\right)\right)+t(-z) \|^{2}
\end{aligned}
$$




$$
\begin{aligned}
& \leq(1-t) \|\left(\mathbb{S} x_{t}-\frac{\tau}{1-t} \mathbb{A} \mathbb{S} x_{t}\right) \\
& \quad-\left(\mathbb{S} z-\frac{\tau}{1-t} \mathbb{A} \mathbb{S} z\right)\left\|^{2}+t\right\| z \|^{2} \\
& \leq(1-t)\left\|x_{t}-z\right\|^{2}+t\|z\|^{2} .
\end{aligned}
$$

Thus,

$$
\begin{aligned}
\left\|x_{t}-z\right\|^{2} \leq \frac{1}{2}( & (1-t)\left\|x_{t}-z\right\|^{2}+t\|z\|^{2}+\left\|x_{t}-z\right\|^{2} \\
& \left.-\left\|(1-t) \mathbb{S} x_{t}-\tau\left(\mathbb{A} \mathbb{S} x_{t}-\mathbb{A} z\right)-x_{t}\right\|^{2}\right) .
\end{aligned}
$$

It follows that

$$
\begin{aligned}
\left\|x_{t}-z\right\|^{2} \leq & (1-t)\left\|x_{t}-z\right\|^{2}+t\|z\|^{2} \\
& -\left\|(1-t) \mathbb{S} x_{t}-x_{t}-\tau\left(\mathbb{A} \mathbb{S} x_{t}-\mathbb{A} z\right)\right\|^{2} \\
= & (1-t)\left\|x_{t}-z\right\|^{2}+t\|z\|^{2}-\left\|(1-t) \mathbb{S} x_{t}-x_{t}\right\|^{2} \\
& +2 \tau\left\langle(1-t) \mathbb{S} x_{t}-x_{t}, \mathbb{A} \mathbb{S} x_{t}-\mathbb{A} z\right\rangle \\
& -\tau^{2}\left\|\mathbb{A} \mathbb{S} x_{t}-\mathbb{A} z\right\|^{2} \\
\leq & (1-t)\left\|x_{t}-z\right\|^{2}+t\|z\|^{2}-\left\|(1-t) \mathbb{S} x_{t}-x_{t}\right\|^{2} \\
& +2 \tau\left\|(1-t) \mathbb{S} x_{t}-x_{t}\right\|\left\|\mathbb{A} \mathbb{S} x_{t}-\mathbb{A} z\right\| .
\end{aligned}
$$

Hence,

$$
\begin{aligned}
& \left\|(1-t) \mathbb{S} x_{t}-x_{t}\right\|^{2} \\
& \quad \leq t\|z\|^{2}+2 \tau\left\|(1-t) \mathbb{S} x_{t}-x_{t}\right\|\left\|\mathbb{A} \mathbb{S} x_{t}-\mathbb{A} z\right\| .
\end{aligned}
$$

This together with (23) implies that

$$
\lim _{t \rightarrow 0+}\left\|(1-t) \mathbb{S} x_{t}-x_{t}\right\|=0 .
$$

So,

$$
\lim _{t \rightarrow 0+}\left\|x_{t}-\mathbb{S} x_{t}\right\|=0 .
$$

By (19), we have

$$
\begin{aligned}
\left\|x_{t}-z\right\|^{2} \leq \|(1-t) & \left(\left(\mathbb{S} x_{t}-\frac{\tau}{1-t} \mathbb{A} \mathbb{S} x_{t}\right)\right. \\
& \left.-\left(z-\frac{\tau}{1-t} \mathbb{A} z\right)\right)+t(-z) \|^{2}
\end{aligned}
$$

$$
\begin{aligned}
& =(1-t)^{2} \|\left(\mathbb{S} x_{t}-\frac{\tau}{1-t} \mathbb{A} \mathbb{S} x_{t}\right) \\
& -\left(z-\frac{\tau}{1-t} \mathbb{A} z\right) \|^{2} \\
& +2 t(1-t)\left\langle-z,\left(\mathbb{S} x_{t}-\frac{\tau}{1-t} \mathbb{A} \mathbb{S} x_{t}\right)\right. \\
& \left.\quad-\left(z-\frac{\tau}{1-t} \mathbb{A} z\right)\right\rangle+t^{2}\|z\|^{2} \\
& \leq(1-t)^{2}\left\|x_{t}-z\right\|^{2}+2 t(1-t) \\
& \quad \times\left\langle-z, \mathbb{S} x_{t}-\frac{\tau}{1-t}\left(\mathbb{A} \mathbb{S} x_{t}-\mathbb{A} \mathbb{S} z\right)-z\right\rangle \\
& +t^{2}\|z\|^{2} .
\end{aligned}
$$

It follows that

$$
\begin{aligned}
\left\|x_{t}-z\right\|^{2} \leq & \left\langle-z, \mathbb{S} x_{t}-\frac{\tau}{1-t}\left(\mathbb{A} \mathbb{S} x_{t}-\mathbb{A} z\right)-z\right\rangle \\
& +\frac{t}{2}\left(\|z\|^{2}+\left\|x_{t}-z\right\|^{2}\right) \\
& +t\|z\|\left\|\mathbb{S} x_{t}-\frac{\tau}{1-t}\left(\mathbb{A} \mathbb{S} x_{t}-\mathbb{A} z\right)-z\right\| \\
\leq & \left\langle-z, \mathbb{S} x_{t}-z\right\rangle+\left(t+\left\|\mathbb{A} \mathbb{S} x_{t}-\mathbb{A} z\right\|\right) M,
\end{aligned}
$$

where $M$ is some constant such that

$$
\begin{aligned}
\sup _{t \in(0,(2 \varrho-\tau) / 2 \varrho)}\left\{\frac{1}{2}\left(\|z\|^{2}+\left\|x_{t}-z\right\|^{2}\right),\right. \\
\left.\|z\|\left\|\mathbb{S} x_{t}-\frac{\tau}{1-t}\left(\mathbb{A} \mathbb{S} x_{t}-\mathbb{A} z\right)-z\right\|\right\} \leq M .
\end{aligned}
$$

Now we show that $\left\{x_{t}\right\}$ is relatively norm-compact as $t \rightarrow 0+$. Assume $\left\{t_{n}\right\} \subset(0,(2 \varrho-\tau) / 2 \varrho)$ such that $t_{n} \rightarrow 0+$ as $n \rightarrow \infty$. Put $x_{n}:=x_{t_{n}}$. From (32), we have

$$
\left\|x_{n}-z\right\|^{2} \leq\left\langle-z, \mathbb{S} x_{n}-z\right\rangle+\left(t_{n}+\left\|\mathbb{A} \mathbb{S} x_{n}-\mathbb{A} z\right\|\right) M .
$$

Since $\left\{x_{n}\right\}$ is bounded, without loss of generality, we may assume that $x_{n_{j}} \rightarrow \tilde{x} \in C$. Hence, $x_{n_{j}}-\left(\tau /\left(1-t_{n_{j}}\right)\right)\left(\mathbb{A} \mathbb{S} x_{n_{j}}-\right.$ $\mathbb{A} z) \rightarrow \tilde{x}$ because of $\left\|\mathbb{A} \mathbb{S} x_{n}-\mathbb{A} z\right\| \rightarrow 0$ by (23). From (30), we have

$$
\lim _{n \rightarrow \infty}\left\|x_{n}-\mathbb{S} x_{n}\right\|=0
$$

By Lemma 5 and (35), we deduce $\tilde{x} \in \operatorname{Fix}(\mathbb{S})$.

Next, we show that $\tilde{x} \in(\mathbb{A}+\mathbb{B})^{-1} 0$. Let $v \in \mathbb{B} u$. Note that $x_{n}=J_{\tau}^{\mathbb{B}}\left(\left(1-t_{n}\right) \mathbb{S} x_{n}-\tau \mathbb{A} \mathbb{S} x_{n}\right)$ for all $n$. Then, we have

$$
\left(1-t_{n}\right) \mathbb{S} x_{n}-\tau \mathbb{A} \mathbb{S} x_{n} \in(I+\tau \mathbb{B}) x_{n} .
$$

So,

$$
\frac{1-t_{n}}{\tau} \mathbb{S} x_{n}-\mathbb{A} \mathbb{S} x_{n}-\frac{x_{n}}{\tau} \in \mathbb{B} x_{n}
$$


Since $\mathbb{B}$ is monotone, we have, for $(u, v) \in \mathbb{B}$,

$$
\begin{aligned}
\left\langle\frac{t_{n} \gamma f\left(x_{n}\right)}{\tau}+\frac{1-t_{n}}{\tau} \mathbb{S} x_{n}-\mathbb{A} \mathbb{S} x_{n}-\frac{x_{n}}{\tau}-v, x_{n}-u\right\rangle \geq 0 \\
\Longrightarrow\left\langle\left(1-t_{n}\right) \mathbb{S} x_{n}-\tau \mathbb{A} \mathbb{S} x_{n}-x_{n}-\tau v, x_{n}-u\right\rangle \geq 0 \\
\Longrightarrow\left\langle\mathbb{A} \mathbb{S} x_{n}+v, x_{n}-u\right\rangle \\
\leq \leq \frac{1}{\tau}\left\langle\mathbb{S} x_{n}-x_{n}, x_{n}-u\right\rangle-\frac{t_{n}}{\tau}\left\langle\mathbb{S} x_{n}, x_{n}-u\right\rangle \\
\Longrightarrow\left\langle\mathbb{A} \mathbb{S} \tilde{x}+v, x_{n}-u\right\rangle \\
\leq \frac{1}{\tau}\left\langle\mathbb{S} x_{n}-x_{n}, x_{n}-u\right\rangle-\frac{t_{n}}{\tau}\left\langle\mathbb{S} x_{n}, x_{n}-u\right\rangle \\
+\left\langle\mathbb{A} \mathbb{S} \tilde{x}-\mathbb{A} \mathbb{S} x_{n}, x_{n}-u\right\rangle \\
\Longrightarrow\left\langle\mathbb{A} \tilde{x}+v, x_{n}-u\right\rangle \\
\leq \frac{1}{\tau}\left\|\mathbb{S} x_{n}-x_{n}\right\|\left\|x_{n}-u\right\|+\frac{t_{n}}{\tau}\left\|\mathbb{S} x_{n}\right\|\left\|x_{n}-u\right\| \\
+\left\|\mathbb{A} \mathbb{S} \tilde{x}-\mathbb{A} \mathbb{S} x_{n}\right\|\left\|x_{n}-u\right\| .
\end{aligned}
$$

It follows that

$$
\begin{aligned}
\langle\mathbb{A} \mathbb{S} \tilde{x}+v, \tilde{x}-u\rangle \leq & \frac{1}{\tau}\left\|\mathbb{S} x_{n_{j}}-x_{n_{j}}\right\|\left\|x_{n_{j}}-u\right\| \\
& +\frac{t_{n_{j}}}{\tau}\left\|\mathbb{S} x_{n_{j}}\right\|\left\|x_{n_{j}}-u\right\| \\
& +\left\|\mathbb{A} \mathbb{S} \tilde{x}-\mathbb{A} \mathbb{S} x_{n_{j}}\right\|\left\|x_{n_{j}}-u\right\| \\
& +\left\langle\mathbb{A} \mathbb{S} \tilde{x}+v, \tilde{x}-x_{n_{j}}\right\rangle .
\end{aligned}
$$

Since

$$
\left\langle x_{n_{j}}-\tilde{x}, \mathbb{A} \mathbb{S} x_{n_{j}}-\mathbb{A} \mathbb{S} \tilde{x}\right\rangle \geq \varrho\left\|\mathbb{A} \mathbb{S} x_{n_{j}}-\mathbb{A} \mathbb{S} \tilde{x}\right\|^{2},
$$

$\mathbb{A} \mathbb{S} x_{n_{j}} \rightarrow \mathbb{A} \mathbb{S} z$, and $x_{n_{j}} \rightarrow \tilde{x}$, we have $\mathbb{A} \mathbb{S} x_{n_{j}} \rightarrow \mathbb{A} \mathbb{S} \tilde{x}$. We also observe that $t_{n} \rightarrow 0$ and $\left\|\mathbb{S} x_{n}-x_{n}\right\| \rightarrow 0$. Then, from (39), we derive

$$
\langle\mathbb{A} \mathbb{S} \tilde{x}+v, \tilde{x}-u\rangle \leq 0 .
$$

That is, $\langle-\mathbb{A} \tilde{x}-v, \tilde{x}-u\rangle \geq 0$. Since $\mathbb{B}$ is maximal monotone, we have $-\mathbb{A} \tilde{x} \in \mathbb{B} \tilde{x}$. This shows that $0 \in(\mathbb{A}+\mathbb{B}) \tilde{x}$. Hence, we have $\tilde{x} \in \operatorname{Fix}(\mathbb{S}) \cap(\mathbb{A}+\mathbb{B})^{-1} 0$. Therefore, we can substitute $\tilde{x}$ for $z$ in (34) to get

$$
\left\|x_{n}-\tilde{x}\right\|^{2} \leq\left\langle-\tilde{x}, \mathbb{S} x_{n}-\tilde{x}\right\rangle+\left(t_{n}+\left\|\mathbb{A} \mathbb{S} x_{n}-\mathbb{A} \tilde{x}\right\|\right) M .
$$

Consequently, the weak convergence of $\left\{x_{n}\right\}$ to $\tilde{x}$ actually implies that $x_{n} \rightarrow \tilde{x}$. This has proved the relative normcompactness of the net $\left\{x_{t}\right\}$ as $t \rightarrow 0+$.

From (34), we get

$$
\|\tilde{x}-z\|^{2} \leq\langle-z, \tilde{x}-z\rangle, \quad \forall z \in \operatorname{Fix}(\mathbb{S}) \cap(\mathbb{A}+\mathbb{B})^{-1} 0 .
$$

That is,

$$
\langle\tilde{x}, \tilde{x}-z\rangle \leq 0, \quad \forall z \in \operatorname{Fix}(\mathbb{S}) \cap(\mathbb{A}+\mathbb{B})^{-1} 0 .
$$

It follows that

$$
\|\tilde{x}\| \leq\|z\|, \quad \forall z \in \operatorname{Fix}(\mathbb{S}) \cap(\mathbb{A}+\mathbb{B})^{-1} 0 .
$$

It is obvious that $\tilde{x}=\operatorname{proj}_{\operatorname{Fix}(\mathbb{S}) \cap(\mathbb{A}+\mathbb{B})^{-1} 0}(0)$ by (44). This denotes that the entire net $\left\{x_{t}\right\}$ converges to $\tilde{x}$. This completes the proof.

Next, we present another algorithm.

Algorithm 9. For given $x_{0} \in \mathscr{C}$, define a sequence $\left\{x_{n}\right\} \subset \mathscr{C}$ iteratively by

$$
\begin{array}{r}
x_{n+1}=\varsigma_{n} x_{n}+\left(1-\varsigma_{n}\right) J_{\tau_{n}}^{\mathbb{B}}\left(\left(1-\varrho_{n}\right) \mathbb{S} x_{n}-\tau_{n} \mathbb{A} \mathbb{S} x_{n}\right), \\
\forall n \geq 0,
\end{array}
$$

where $\left\{\tau_{n}\right\} \subset(0,2 \varrho),\left\{\varrho_{n}\right\} \subset(0,1)$, and $\left\{\varsigma_{n}\right\} \subset(0,1)$.

Theorem 10. Suppose that $\operatorname{Fix}(\mathbb{S}) \cap(\mathbb{A}+\mathbb{B})^{-1} 0 \neq \emptyset$. Assume that the following conditions are satisfied:

(i) $\lim _{n \rightarrow \infty} \varrho_{n}=0$ and $\sum_{n} \varrho_{n}=\infty$;

(ii) $0<\underline{\lim }_{n \rightarrow \infty} \varsigma_{n} \leq \overline{\lim }_{n \rightarrow \infty} \varsigma_{n}<1$;

(iii) $a\left(1-\varrho_{n}\right) \leq \tau_{n} \leq b\left(1-\varrho_{n}\right)$, where $[a, b] \subset(0,2 \varrho)$ and $\lim _{n \rightarrow \infty}\left(\tau_{n+1}-\tau_{n}\right)=0$.

Then $\left\{x_{n}\right\}$ generated by (46) converges strongly to a point $\tilde{x}=$ $\operatorname{proj}_{\operatorname{Fix}(\mathbb{S}) \cap(\mathbb{A}+\mathbb{B})^{-1}(0)}(0)$ which is the minimum norm element in $\operatorname{Fix}(\mathbb{S}) \cap(\mathbb{A}+\mathbb{B})^{-1}(0)$.

Proof. Let $z \in \operatorname{Fix}(\mathbb{S}) \cap(\mathbb{A}+\mathbb{B})^{-1}(0)$. We have $z=J_{\tau_{n}}^{\mathbb{B}}(z-$ $\left.\tau_{n} \mathbb{A} z\right)=J_{\tau_{n}}^{\mathbb{B}}\left(\varrho_{n} z+\left(1-\varrho_{n}\right)\left(z-\tau_{n} \mathbb{A} z /\left(1-\varrho_{n}\right)\right)\right)$ for all $n \geq 0$. Since $J_{\tau_{n}}^{\mathbb{B}}, \mathbb{S}$, and $I-\tau_{n} \mathbb{A} /\left(1-\varrho_{n}\right)$ are nonexpansive, we have

$$
\begin{aligned}
\left\|J_{\tau_{n}}^{\mathbb{B}}\left(\left(1-\varrho_{n}\right) \mathbb{S} x_{n}-\tau_{n} \mathbb{A} \mathbb{S} x_{n}\right)-z\right\| & \| J_{\tau_{n}}^{\mathbb{B}}\left(\left(1-\varrho_{n}\right)\left(\mathbb{S} x_{n}-\frac{\tau_{n}}{1-\varrho_{n}} \mathbb{A} \mathbb{S} x_{n}\right)\right) \\
& -J_{\tau_{n}}^{\mathbb{B}}\left(\varrho_{n} z+\left(1-\varrho_{n}\right)\left(z-\frac{\tau_{n}}{1-\varrho_{n}} \mathbb{A} z\right)\right) \| \\
\leq & \|\left(\left(1-\varrho_{n}\right)\left(\mathbb{S} x_{n}-\frac{\tau_{n}}{1-\varrho_{n}} \mathbb{A} \mathbb{S} x_{n}\right)\right) \\
& -\left(\varrho_{n} z+\left(1-\varrho_{n}\right)\left(z-\frac{\tau_{n}}{1-\varrho_{n}} \mathbb{A} z\right)\right) \| \\
= & \|\left(1-\varrho_{n}\right)\left(\mathbb{S} x_{n}-\frac{\tau_{n}}{1-\varrho_{n}} \mathbb{A} \mathbb{S} x_{n}\right. \\
\leq & \left(1-\left(z-\frac{\tau_{n}}{1-\varrho_{n}} \mathbb{A} z\right)\right)+\varrho_{n}(-z) \|
\end{aligned}
$$


6

Abstract and Applied Analysis

Thus,

$$
\begin{aligned}
\left\|x_{n+1}-z\right\| \leq & \varsigma_{n}\left\|x_{n}-z\right\|+\left(1-\varsigma_{n}\right)\left(1-\varrho_{n}\right)\left\|x_{n}-z\right\| \\
& +\left(1-\varsigma_{n}\right) \varrho_{n}\|z\| \\
= & {\left[1-\varrho_{n}\left(1-\varsigma_{n}\right)\right]\left\|x_{n}-z\right\|+\left(1-\varsigma_{n}\right) \varrho_{n}\|z\| . }
\end{aligned}
$$

By induction, we have

$$
\left\|x_{n+1}-z\right\| \leq \max \left\{\left\|x_{0}-z\right\|,\|z\|\right\} .
$$

Therefore, $\left\{x_{n}\right\}$ is bounded.

From (4) and (47), we derive

$$
\begin{aligned}
\| & \left(1-\varrho_{n}\right)\left(\left(\mathbb{S} x_{n}-\frac{\tau_{n}}{1-\varrho_{n}} \mathbb{A} \mathbb{S} x_{n}\right)-\left(z-\frac{\tau_{n}}{1-\varrho_{n}} \mathbb{A} z\right)\right) \\
& +\varrho_{n}(-z)\|\|^{2} \\
\leq & \left(1-\varrho_{n}\right)\left\|\left(\mathbb{S} x_{n}-\frac{\tau_{n}}{1-\varrho_{n}} \mathbb{A} \mathbb{S} x_{n}\right)-\left(z-\frac{\tau_{n}}{1-\varrho_{n}} \mathbb{A} z\right)\right\|^{2} \\
& +\varrho_{n}\|z\|^{2} \quad\left(1-\varrho_{n}\right)\left\|\left(\mathbb{S} x_{n}-z\right)-\frac{\tau_{n}}{1-\varrho_{n}}\left(\mathbb{A} \mathbb{S} x_{n}-A z\right)\right\|^{2}+\varrho_{n}\|z\|^{2} \\
= & \left(1-\varrho_{n}\right)\left(\left\|\mathbb{S} x_{n}-z\right\|^{2}-\frac{2 \tau_{n}}{1-\varrho_{n}}\left\langle\mathbb{A} \mathbb{S} x_{n}-\mathbb{A} z, \mathbb{S} x_{n}-z\right\rangle\right. \\
= & \left.+\frac{\tau_{n}^{2}}{\left(1-\varrho_{n}\right)^{2}}\left\|\mathbb{A} \mathbb{S} x_{n}-\mathbb{A} z\right\|^{2}\right)+\varrho_{n}\|z\|^{2} \\
& \left.\quad+\frac{\tau_{n}^{2}}{\left(1-\varrho_{n}\right)^{2}}\left\|\mathbb{A} \mathbb{S} x_{n}-\mathbb{A} z\right\|^{2}\right)+\varrho_{n}\|z\|^{2} \\
\leq & \left(1-\varrho_{n}\right)\left(\left\|x_{n}-z\right\|^{2}-\frac{2 \varrho \tau_{n}}{1-\varrho_{n}}\left\|\mathbb{A} \mathbb{S} x_{n}-\mathbb{A} z\right\|^{2}\right.
\end{aligned}
$$

Set $u_{n}=\left(1-\varrho_{n}\right) \mathbb{S} x_{n}-\tau_{n} \mathbb{A} \mathbb{S} x_{n}$ for all $n \geq 0$. Since $\tau_{n}-2(1-$ $\left.\varrho_{n}\right) \varrho \leq 0$ for all $n \geq 0$, we obtain

$$
\begin{gathered}
\left\|J_{\tau_{n}}^{\mathbb{B}} u_{n}-z\right\|^{2} \\
\leq\left(1-\varrho_{n}\right)\left(\left\|x_{n}-z\right\|^{2}+\frac{\tau_{n}}{\left(1-\varrho_{n}\right)^{2}}\left(\tau_{n}-2\left(1-\varrho_{n}\right) \varrho\right)\right. \\
\left.\times\left\|\mathbb{A} \mathbb{S} x_{n}-\mathbb{A} z\right\|^{2}\right)+\varrho_{n}\|z\|^{2} .
\end{gathered}
$$

From (46), we have

$$
\begin{aligned}
\left\|x_{n+1}-z\right\|^{2} & =\left\|\varsigma_{n}\left(x_{n}-z\right)+\left(1-\varsigma_{n}\right)\left(J_{\tau_{n}}^{\mathbb{B}} u_{n}-z\right)\right\|^{2} \\
& \leq \varsigma_{n}\left\|x_{n}-z\right\|^{2}+\left(1-\varsigma_{n}\right)\left\|J_{\tau_{n}}^{\mathbb{B}} u_{n}-z\right\|^{2} .
\end{aligned}
$$

Set $y_{n}=J_{\tau_{n}}^{\mathbb{B}}\left(\left(1-\varrho_{n}\right) \mathbb{S} x_{n}-\tau_{n} \mathbb{A} \mathbb{S} x_{n}\right)$ for all $n \geq 0$. Then $x_{n+1}=$ $\varsigma_{n} x_{n}+\left(1-\varsigma_{n}\right) y_{n}$ for all $n \geq 0$. Next, we estimate $\left\|x_{n+1}-x_{n}\right\|$. In fact, we have

$$
\begin{aligned}
\left\|y_{n+1}-y_{n}\right\|= & \left\|J_{\tau_{n+1}}^{\mathbb{B}} u_{n+1}-J_{\tau_{n}}^{\mathbb{B}} u_{n}\right\| \\
\leq & \left\|J_{\tau_{n+1}}^{\mathbb{B}} u_{n+1}-J_{\tau_{n+1}}^{\mathbb{B}} u_{n}\right\|+\left\|J_{\tau_{n+1}}^{\mathbb{B}} u_{n}-J_{\tau_{n}}^{\mathbb{B}} u_{n}\right\| \\
\leq & \|\left(\left(1-\varrho_{n+1}\right) \mathbb{S} x_{n+1}-\tau_{n+1} \mathbb{A} \mathbb{S} x_{n+1}\right) \\
& -\left(\left(1-\varrho_{n}\right) \mathbb{S} x_{n}-\tau_{n} \mathbb{A} \mathbb{S} x_{n}\right) \| \\
& +\left\|J_{\tau_{n+1}}^{\mathbb{B}} u_{n}-J_{\tau_{n}}^{\mathbb{B}} u_{n}\right\| \\
= & \|\left(I-\tau_{n+1} \mathbb{A}\right) \mathbb{S} x_{n+1}-\left(I-\tau_{n+1} \mathbb{A}\right) \mathbb{S} x_{n} \\
& +\left(\tau_{n}-\tau_{n+1}\right) \mathbb{A} \mathbb{S} x_{n}+\varrho_{n} \mathbb{S} x_{n}-\varrho_{n+1} \mathbb{S} x_{n+1} \| \\
& +\left\|J_{\tau_{n+1}}^{\mathbb{B}} u_{n}-J_{\tau_{n}}^{\mathbb{B}} u_{n}\right\| \\
\leq & \left\|\left(I-\tau_{n+1} \mathbb{A}\right) \mathbb{S} x_{n+1}-\left(I-\tau_{n+1} \mathbb{A}\right) \mathbb{S} x_{n}\right\| \\
& +\left|\tau_{n+1}-\tau_{n}\right|\left\|\mathbb{A} \mathbb{S} x_{n}\right\|+\varrho_{n}\left\|\mathbb{S} x_{n}\right\| \\
& +\varrho_{n+1}\left\|\mathbb{S} x_{n+1}\right\|+\left\|J_{\tau_{n+1}}^{\mathbb{B}} u_{n}-J_{\tau_{n}}^{\mathbb{B}} u_{n}\right\| .
\end{aligned}
$$

Since $I-\tau_{n+1} A$ is nonexpansive for $\tau_{n+1} \in(0,2 \varrho)$, we have

$$
\begin{gathered}
\left\|\left(I-\tau_{n+1} \mathbb{A}\right) \mathbb{S} x_{n+1}-\left(I-\tau_{n+1} \mathbb{A}\right) \mathbb{S} x_{n}\right\| \\
\leq\left\|\mathbb{S} x_{n+1}-\mathbb{S} x_{n}\right\| \leq\left\|x_{n+1}-x_{n}\right\| .
\end{gathered}
$$

From (13), we have

$$
J_{\tau_{n+1}}^{\mathbb{B}} u_{n}=J_{\tau_{n}}^{\mathbb{B}}\left(\frac{\tau_{n}}{\tau_{n+1}} u_{n}+\left(1-\frac{\tau_{n}}{\tau_{n+1}}\right) J_{\tau_{n+1}}^{\mathbb{B}} u_{n}\right) .
$$

It follows that

$$
\begin{aligned}
& \left\|J_{\tau_{n+1}}^{\mathbb{B}} u_{n}-J_{\tau_{n}}^{\mathbb{B}} u_{n}\right\| \\
& \quad=\left\|J_{\tau_{n}}^{\mathbb{B}}\left(\frac{\tau_{n}}{\tau_{n+1}} u_{n}+\left(1-\frac{\tau_{n}}{\tau_{n+1}}\right) J_{\tau_{n+1}}^{\mathbb{B}} u_{n}\right)-J_{\tau_{n}}^{\mathbb{B}} u_{n}\right\| \\
& \quad \leq\left\|\left(\frac{\tau_{n}}{\tau_{n+1}} u_{n}+\left(1-\frac{\tau_{n}}{\tau_{n+1}}\right) J_{\tau_{n+1}}^{\mathbb{B}} u_{n}\right)-u_{n}\right\| \\
& \quad \leq \frac{\left|\tau_{n+1}-\tau_{n}\right|}{\tau_{n+1}}\left\|u_{n}-J_{\tau_{n+1}}^{\mathbb{B}} u_{n}\right\| .
\end{aligned}
$$


So,

$$
\begin{aligned}
\left\|y_{n+1}-y_{n}\right\| \leq & \left\|x_{n+1}-x_{n}\right\|+\left|\tau_{n+1}-\tau_{n}\right|\left\|\mathbb{A} S x_{n}\right\| \\
& +\varrho_{n}\left\|\mathbb{S} x_{n}\right\| \\
& +\varrho_{n+1}\left\|\mathbb{S} x_{n+1}\right\|+\frac{\left|\tau_{n+1}-\tau_{n}\right|}{\tau_{n+1}}\left\|u_{n}-J_{\tau_{n+1}}^{\mathbb{B}} u_{n}\right\| .
\end{aligned}
$$

Then,

$$
\begin{aligned}
& \left\|y_{n+1}-y_{n}\right\|-\left\|x_{n+1}-x_{n}\right\| \\
& \quad \leq\left|\tau_{n+1}-\tau_{n}\right|\left\|\mathbb{A} S x_{n}\right\|+\varrho_{n}\left\|\mathbb{S} x_{n}\right\| \\
& \quad+\varrho_{n+1}\left\|\mathbb{S} x_{n+1}\right\|+\frac{\left|\tau_{n+1}-\tau_{n}\right|}{\tau_{n+1}}\left\|u_{n}-J_{\tau_{n+1}}^{\mathbb{B}} u_{n}\right\| .
\end{aligned}
$$

Since $\varrho_{n} \rightarrow 0, \tau_{n+1}-\tau_{n} \rightarrow 0$ and $\underline{\lim }_{n \rightarrow \infty} \tau_{n}>0$, we obtain

$$
\limsup _{n \rightarrow \infty}\left(\left\|y_{n+1}-y_{n}\right\|-\left\|x_{n+1}-x_{n}\right\|\right) \leq 0 .
$$

By Lemma 6, we get

$$
\lim _{n \rightarrow \infty}\left\|y_{n}-x_{n}\right\|=0
$$

Consequently, we obtain

$$
\lim _{n \rightarrow \infty}\left\|x_{n+1}-x_{n}\right\|=\lim _{n \rightarrow \infty}\left(1-\varsigma_{n}\right)\left\|y_{n}-x_{n}\right\|=0 .
$$

From (51) and (52), we have

$$
\begin{aligned}
\left\|x_{n+1}-z\right\|^{2} \leq & \varsigma_{n}\left\|x_{n}-z\right\|^{2}+\left(1-\varsigma_{n}\right)\left\|{\mathcal{\tau _ { n }}}_{n}^{\mathbb{B}} u_{n}-z\right\|^{2} \\
\leq & \left(1-\varsigma_{n}\right)\left(1-\varrho_{n}\right) \\
& \times\left(\left\|x_{n}-z\right\|^{2}+\frac{\tau_{n}}{\left(1-\varrho_{n}\right)^{2}}\left(\tau_{n}-2\left(1-\varrho_{n}\right) \varrho\right)\right. \\
& \left.\quad \times\left\|\mathbb{A} \mathbb{S} x_{n}-\mathbb{A} z\right\|^{2}\right) \\
& +\left(1-\varsigma_{n}\right) \varrho_{n}\|z\|^{2}+\varsigma_{n}\left\|x_{n}-z\right\|^{2} \\
= & {\left[1-\left(1-\varsigma_{n}\right) \varrho_{n}\right]\left\|x_{n}-z\right\|^{2} } \\
& +\frac{\left(1-\varsigma_{n}\right) \tau_{n}}{1-\varrho_{n}}\left(\tau_{n}-2\left(1-\varrho_{n}\right) \varrho\right)\left\|\mathbb{A} \mathbb{S} x_{n}-\mathbb{A} z\right\|^{2} \\
& +\left(1-\varsigma_{n}\right) \varrho_{n}\|z\|^{2} \\
\leq & \left\|x_{n}-z\right\|^{2}+\frac{\left(1-\varsigma_{n}\right) \tau_{n}}{1-\varrho_{n}}\left(\tau_{n}-2\left(1-\varrho_{n}\right) \varrho\right) \\
& \times\left\|\mathbb{A S S} x_{n}-\mathbb{A} z\right\|^{2}+\left(1-\varsigma_{n}\right) \varrho_{n}\|z\|^{2} .
\end{aligned}
$$

Then, we obtain

$$
\begin{aligned}
& \frac{\left(1-\varsigma_{n}\right) \tau_{n}}{\left(1-\varrho_{n}\right)}\left(2\left(1-\varrho_{n}\right) \varrho-\tau_{n}\right)\left\|\mathbb{A} \mathbb{S} x_{n}-\mathbb{A} z\right\|^{2} \\
& \leq\left\|x_{n}-z\right\|^{2}-\left\|x_{n+1}-z\right\|^{2}+\left(1-\varsigma_{n}\right) \varrho_{n}\|z\|^{2} \\
& \leq\left(\left\|x_{n}-z\right\|-\left\|x_{n+1}-z\right\|\right)\left\|x_{n+1}-x_{n}\right\| \\
& \quad+\left(1-\varsigma_{n}\right) \varrho_{n}\|z\|^{2} .
\end{aligned}
$$

Since $\lim _{n \rightarrow \infty} \varrho_{n}=0, \lim _{n \rightarrow \infty}\left\|x_{n+1}-x_{n}\right\|=0$, and $\underline{\lim }_{n \rightarrow \infty}\left(\left(1-\varsigma_{n}\right) \tau_{n} /\left(1-\varrho_{n}\right)\right)\left(2\left(1-\varrho_{n}\right) \varrho-\tau_{n}\right)>0$, we have

$$
\lim _{n \rightarrow \infty}\left\|\mathbb{A} \mathbb{S} x_{n}-\mathbb{A} z\right\|=0 .
$$

Next, we show $\left\|x_{n}-\mathbb{S} x_{n}\right\| \rightarrow 0$. By using the firm nonexpansivity of $J_{\tau_{n}}^{\mathbb{B}}$, we have

$$
\begin{aligned}
\| J_{\tau_{n}}^{\mathbb{B}} & u_{n}-z \|^{2} \\
= & \left\|J_{\tau_{n}}^{\mathbb{B}}\left(\left(1-\varrho_{n}\right) \mathbb{S} x_{n}-\tau_{n} \mathbb{A} \mathbb{S} x_{n}\right)-J_{\tau_{n}}^{\mathbb{B}}\left(z-\tau_{n} \mathbb{A} z\right)\right\|^{2} \\
\leq & \left\langle\left(1-\varrho_{n}\right) \mathbb{S} x_{n}-\tau_{n} \mathbb{A} \mathbb{S} x_{n}-\left(z-\tau_{n} \mathbb{A} z\right), J_{\tau_{n}}^{\mathbb{B}} u_{n}-z\right\rangle \\
= & \frac{1}{2}\left(\left\|\left(1-\varrho_{n}\right) \mathbb{S} x_{n}-\tau_{n} \mathbb{A} \mathbb{S} x_{n}-\left(z-\tau_{n} \mathbb{A} z\right)\right\|^{2}\right. \\
& \quad\left\|J_{\tau_{n}}^{\mathbb{B}} u_{n}-z\right\|^{2} \\
& \left.\quad\left\|\left(1-\varrho_{n}\right) \mathbb{S} x_{n}-\tau_{n}\left(\mathbb{A} \mathbb{S} x_{n}-\mathbb{A} z\right)-J_{\tau_{n}}^{\mathbb{B}} u_{n}\right\|^{2}\right) .
\end{aligned}
$$

Observe that

$$
\begin{aligned}
& \left\|\left(1-\varrho_{n}\right) \mathbb{S} x_{n}-\tau_{n} \mathbb{A} \mathbb{S} x_{n}-\left(z-\tau_{n} \mathbb{A} z\right)\right\|^{2} \\
& =\|\left(1-\varrho_{n}\right)\left(\mathbb{S} x_{n}-\frac{\tau_{n}}{1-\varrho_{n}} \mathbb{A} \mathbb{S} x_{n}\right. \\
& \left.\quad-\left(z-\frac{\tau_{n}}{1-\varrho_{n}} \mathbb{A} z\right)\right)+\varrho_{n}(-z) \|^{2} \\
& \leq\left(1-\varrho_{n}\right) \| \mathbb{S} x_{n}-\frac{\tau_{n}}{1-\varrho_{n}} \mathbb{A} \mathbb{S} x_{n} \\
& \quad-\left(z-\frac{\tau_{n}}{1-\varrho_{n}} \mathbb{A} z\right)\left\|^{2}+\varrho_{n}\right\| z \|^{2} \\
& \leq\left(1-\varrho_{n}\right)\left\|x_{n}-z\right\|^{2}+\varrho_{n}\|z\|^{2} .
\end{aligned}
$$

Hence,

$$
\begin{aligned}
\left\|J_{\tau_{n}}^{\mathbb{B}} u_{n}-z\right\|^{2} \leq \frac{1}{2} & \left(\left(1-\varrho_{n}\right)\left\|x_{n}-z\right\|^{2}+\varrho_{n}\|z\|^{2}+\left\|J_{\tau_{n}}^{\mathbb{B}} u_{n}-z\right\|^{2}\right. \\
& \left.-\left\|\left(1-\varrho_{n}\right) \mathbb{S} x_{n}-J_{\tau_{n}}^{\mathbb{B}} u_{n}-\tau_{n}\left(\mathbb{A} \mathbb{S} x_{n}-\mathbb{A} z\right)\right\|^{2}\right) .
\end{aligned}
$$


It follows that

$$
\begin{aligned}
\left\|J_{\tau_{n}}^{\mathbb{B}} u_{n}-z\right\|^{2} \leq & \left(1-\varrho_{n}\right)\left\|x_{n}-z\right\|^{2}+\varrho_{n}\|z\|^{2} \\
& -\left\|\left(1-\varrho_{n}\right) \mathbb{S} x_{n}-J_{\tau_{n}}^{\mathbb{B}} u_{n}-\tau_{n}\left(\mathbb{A} \mathbb{S} x_{n}-\mathbb{A} z\right)\right\|^{2} \\
= & \left(1-\varrho_{n}\right)\left\|x_{n}-z\right\|^{2}+\varrho_{n}\|z\|^{2} \\
& -\left\|\left(1-\varrho_{n}\right) \mathbb{S} x_{n}-J_{\tau_{n}}^{\mathbb{B}} u_{n}\right\|^{2} \\
& +2 \tau_{n}\left\langle\left(1-\varrho_{n}\right) \mathbb{S} x_{n}-J_{\tau_{n}}^{\mathbb{B}} u_{n}, \mathbb{A} \mathbb{S} x_{n}-\mathbb{A} z\right\rangle \\
& -\tau_{n}^{2}\left\|\mathbb{A} \mathbb{S} x_{n}-\mathbb{A} z\right\|^{2} \\
\leq & \left(1-\varrho_{n}\right)\left\|x_{n}-z\right\|^{2}+\varrho_{n}\|z\|^{2} \\
& -\left\|\left(1-\varrho_{n}\right) \mathbb{S} x_{n}-J_{\tau_{n}}^{\mathbb{B}} u_{n}\right\|^{2} \\
& +2 \tau_{n}\left\|\left(1-\varrho_{n}\right) \mathbb{S} x_{n}-J_{\tau_{n}}^{\mathbb{B}} u_{n}\right\|\left\|\mathbb{A} \mathbb{S} x_{n}-\mathbb{A} z\right\| .
\end{aligned}
$$

This together with (52) implies that

$$
\begin{aligned}
\left\|x_{n+1}-z\right\|^{2} \leq & \varsigma_{n}\left\|x_{n}-z\right\|^{2}+\left(1-\varsigma_{n}\right)\left(1-\varrho_{n}\right)\left\|x_{n}-z\right\|^{2} \\
& +\left(1-\varsigma_{n}\right) \varrho_{n}\|z\|^{2} \\
& -\left(1-\varsigma_{n}\right)\left\|\left(1-\varrho_{n}\right) \mathbb{S} x_{n}-J_{\tau_{n}}^{\mathbb{B}} u_{n}\right\|^{2} \\
& +2 \tau_{n}\left(1-\varsigma_{n}\right)\left\|\left(1-\varrho_{n}\right) \mathbb{S} x_{n}-J_{\tau_{n}}^{\mathbb{B}} u_{n}\right\| \\
& \times\left\|\mathbb{A} \mathbb{S} x_{n}-\mathbb{A} z\right\| \\
= & {\left[1-\left(1-\varsigma_{n}\right) \varrho_{n}\right]\left\|x_{n}-z\right\|^{2}+\left(1-\varsigma_{n}\right) \varrho_{n}\|z\|^{2} } \\
& -\left(1-\varsigma_{n}\right)\left\|\left(1-\varrho_{n}\right) \mathbb{S} x_{n}-J_{\tau_{n}}^{\mathbb{B}} u_{n}\right\|^{2} \\
& +2 \tau_{n}\left(1-\varsigma_{n}\right)\left\|\left(1-\varrho_{n}\right) \mathbb{S} x_{n}-J_{\tau_{n}}^{\mathbb{B}} u_{n}\right\| \\
& \times\left\|\mathbb{A} \mathbb{S} x_{n}-\mathbb{A} z\right\| .
\end{aligned}
$$

Hence,

$$
\begin{aligned}
\left(1-\varsigma_{n}\right) & \left\|\left(1-\varrho_{n}\right) \mathbb{S} x_{n}-J_{\tau_{n}}^{\mathbb{B}} u_{n}\right\|^{2} \\
\leq & \left\|x_{n}-z\right\|^{2}-\left\|x_{n+1}-z\right\|^{2}-\left(1-\varsigma_{n}\right) \varrho_{n}\left\|x_{n}-z\right\|^{2} \\
& +\left(1-\varsigma_{n}\right) \varrho_{n}\|z\|^{2}+2 \tau_{n}\left(1-\varsigma_{n}\right) \\
& \times\left\|\left(1-\varrho_{n}\right) \mathbb{S} x_{n}-J_{\tau_{n}}^{\mathbb{B}} u_{n}\right\|\left\|\mathbb{A} \mathbb{S} x_{n}-\mathbb{A} z\right\| \\
\leq & \left(\left\|x_{n}-z\right\|+\left\|x_{n+1}-z\right\|\right)\left\|x_{n+1}-x_{n}\right\| \\
& +\left(1-\varsigma_{n}\right) \varrho_{n}\|z\|^{2}+2 \tau_{n}\left(1-\varsigma_{n}\right) \\
& \times\left\|\left(1-\varrho_{n}\right) \mathbb{S} x_{n}-J_{\tau_{n}}^{\mathbb{B}} u_{n}\right\|\left\|\mathbb{A} \mathbb{S} x_{n}-\mathbb{A} z\right\| .
\end{aligned}
$$

Since $\overline{\lim }_{n \rightarrow \infty} \varsigma_{n}<1,\left\|x_{n+1}-x_{n}\right\| \rightarrow 0, \varrho_{n} \rightarrow 0$, and $\| \mathbb{A} \mathbb{S} x_{n}-$ $A z \| \rightarrow 0$ (by (60)), we deduce

$$
\lim _{n \rightarrow \infty}\left\|\left(1-\varrho_{n}\right) \mathbb{S} x_{n}-J_{\tau_{n}}^{\mathbb{B}} u_{n}\right\|=0
$$

This indicates that

$$
\lim _{n \rightarrow \infty}\left\|\mathbb{S} x_{n}-J_{\tau_{n}}^{\mathbb{B}} u_{n}\right\|=0
$$

Combining (60) and (72), we get

$$
\lim _{n \rightarrow \infty}\left\|x_{n}-\mathbb{S} x_{n}\right\|=0
$$

Put $\tilde{x}=\lim _{t \rightarrow 0+} x_{t}=\operatorname{proj}_{\operatorname{Fix}(\mathbb{S}) \cap(\mathbb{A}+\mathbb{B})^{-1}(0)}(0)$, where $x_{t}$ is the net defined by (17). We will finally show that $x_{n} \rightarrow \tilde{x}$.

Set $v_{n}=x_{n}-\left(\tau_{n} /\left(1-\varrho_{n}\right)\right)\left(\mathbb{A} \mathbb{S} x_{n}-\mathbb{A} \tilde{x}\right)$ for all $n$. Take $z=\tilde{x}$ in (64) to get $\left\|\mathbb{A} \mathbb{S} x_{n}-\mathbb{A} \tilde{x}\right\| \rightarrow 0$. First, we prove $\overline{\lim }_{n \rightarrow \infty}\left\langle-\tilde{x}, \mathbb{S} x_{n}-\tilde{x}\right\rangle \leq 0$. We take a subsequence $\left\{\mathbb{S} x_{n_{i}}\right\}$ of $\left\{\mathbb{S} x_{n}\right\}$ such that

$$
\varlimsup_{n \rightarrow \infty}\left\langle-\tilde{x}, \mathbb{S} x_{n}-\tilde{x}\right\rangle=\lim _{i \rightarrow \infty}\left\langle-\tilde{x}, \mathbb{S} x_{n_{i}}-\tilde{x}\right\rangle
$$

It is clear that $\left\{\mathbb{S} x_{n_{i}}\right\}$ is bounded due to the boundedness of $\left\{\mathbb{S} x_{n}\right\}$ and $\left\|\mathbb{A} \mathbb{S} x_{n}-\mathbb{A} \tilde{x}\right\| \rightarrow 0$. Then, there exists a subsequence $\left\{\mathbb{S} x_{n_{i_{j}}}\right\}$ of $\left\{\mathbb{S} x_{n_{i}}\right\}$ which converges weakly to some point $w \in \mathscr{C}$. Hence, $\left\{x_{n_{i_{j}}}\right\}$ and $\left\{y_{n_{i_{j}}}\right\}$ also converge weakly to $w$ because of $\left\|\mathbb{S} x_{n_{i_{j}}}-x_{n_{i_{j}}}\right\| \rightarrow 0$ and $\left\|x_{n_{i_{j}}}-y_{n_{i_{j}}}\right\| \rightarrow$ 0 . By the demiclosedness principle of the nonexpansive mapping (see Lemma 5) and (73), we deduce $w \in \operatorname{Fix}(\mathbb{S})$. Furthermore, by similar argument as that of Theorem 8 , we can show that $w$ is also in $(\mathbb{A}+\mathbb{B})^{-1}(0)$. Hence, we have $w \in \operatorname{Fix}(\mathbb{S}) \cap(\mathbb{A}+\mathbb{B})^{-1}(0)$. This implies that

$$
\begin{aligned}
\varlimsup_{n \rightarrow \infty}\left\langle-\tilde{x}, \mathbb{S} x_{n}-\tilde{x}\right\rangle & =\lim _{j \rightarrow \infty}\left\langle-\tilde{x}, \mathbb{S} x_{n_{i_{j}}}-\tilde{x}\right\rangle \\
& =\langle-\tilde{x}, w-\tilde{x}\rangle .
\end{aligned}
$$

Note that $\tilde{x}=\operatorname{proj}_{\operatorname{Fix}(\mathbb{S}) \cap(\mathbb{A}+\mathbb{B})^{-1}(0)}(0)$. Then, $\langle-\tilde{x}, w-\tilde{x}\rangle \leq$ $0, w \in \operatorname{Fix}(\mathbb{S}) \cap(\mathbb{A}+\mathbb{B})^{-1}(0)$. Therefore,

$$
\varlimsup_{n \rightarrow \infty}\left\langle-\tilde{x}, \mathbb{S} x_{n}-\tilde{x}\right\rangle \leq 0
$$

From (46), we have

$$
\begin{aligned}
& \left\|x_{n+1}-\tilde{x}\right\|^{2} \\
& \quad \leq \varsigma_{n}\left\|x_{n}-\tilde{x}\right\|^{2}+\left(1-\varsigma_{n}\right)\left\|J_{\tau_{n}}^{\mathbb{B}} u_{n}-\tilde{x}\right\|^{2} \\
& \quad=\varsigma_{n}\left\|x_{n}-\tilde{x}\right\|^{2}+\left(1-\varsigma_{n}\right)\left\|J_{\tau_{n}}^{\mathbb{B}} u_{n}-J_{\tau_{n}}^{\mathbb{B}}\left(\tilde{x}-\tau_{n} A \tilde{x}\right)\right\|^{2} \\
& \quad \leq \varsigma_{n}\left\|x_{n}-\tilde{x}\right\|^{2}+\left(1-\varsigma_{n}\right)\left\|u_{n}-\left(\tilde{x}-\tau_{n} \mathbb{A} \tilde{x}\right)\right\|^{2}
\end{aligned}
$$




$$
\begin{aligned}
& =\varsigma_{n}\left\|x_{n}-\tilde{x}\right\|^{2}+\left(1-\varsigma_{n}\right) \\
& \times\left\|\left(1-\varrho_{n}\right) \mathbb{S} x_{n}-\tau_{n} \mathbb{A} \mathbb{S} x_{n}-\left(\tilde{x}-\tau_{n} \mathrm{~A} \tilde{x}\right)\right\|^{2} \\
& =\left(1-\varsigma_{n}\right) \|\left(1-\varrho_{n}\right)\left(\left(\mathbb{S} x_{n}-\frac{\tau_{n}}{1-\varrho_{n}} \mathbb{A} \mathbb{S} x_{n}\right)\right. \\
& \left.-\left(\tilde{x}-\frac{\tau_{n}}{1-\varrho_{n}} \AA \tilde{x}\right)\right) \\
& +\varrho_{n}(-\widetilde{x})\left\|^{2}+\varsigma_{n}\right\| x_{n}-\tilde{x} \|^{2} \\
& =\varsigma_{n}\left\|x_{n}-\tilde{x}\right\|^{2}+\left(1-\varsigma_{n}\right) \\
& \times\left(\left(1-\varrho_{n}\right)^{2} \|\left(\mathbb{S} x_{n}-\frac{\tau_{n}}{1-\varrho_{n}} \mathbb{A} \mathbb{S} x_{n}\right)\right. \\
& -\left(\tilde{x}-\frac{\tau_{n}}{1-\varrho_{n}} A \tilde{x}\right) \|^{2} \\
& +2 \varrho_{n}\left(1-\varrho_{n}\right)\left\langle-\tilde{x},\left(\mathbb{S} x_{n}-\frac{\tau_{n}}{1-\varrho_{n}} \mathbb{A} \mathbb{S} x_{n}\right)\right. \\
& \left.\left.-\left(\tilde{x}-\frac{\tau_{n}}{1-\varrho_{n}} \mathbb{A} \tilde{x}\right)\right\rangle+\varrho_{n}^{2}\|\tilde{x}\|^{2}\right) \\
& \leq \varsigma_{n}\left\|x_{n}-\tilde{x}\right\|^{2}+\left(1-c_{n}\right) \\
& \times\left(\left(1-\varrho_{n}\right)^{2}\left\|x_{n}-\tilde{x}\right\|^{2}+2 \varrho_{n} \tau_{n}\left\langle-\tilde{x}, \mathbb{A} \mathbb{S} x_{n}-\mathbb{A} \tilde{x}\right\rangle\right. \\
& \left.+2 \varrho_{n}\left(1-\varrho_{n}\right)\left\langle-\tilde{x}, \mathbb{S} x_{n}-\tilde{x}\right\rangle+\varrho_{n}^{2}\|\tilde{x}\|^{2}\right) \\
& \leq \varsigma_{n}\left\|x_{n}-\tilde{x}\right\|^{2}+\left(1-\varsigma_{n}\right) \\
& \times\left(\left(1-\varrho_{n}\right)^{2}\left\|x_{n}-\tilde{x}\right\|^{2}+2 \varrho_{n} \tau_{n}\|\tilde{x}\|\left\|\mathbb{A} \mathbb{S} x_{n}-\mathbb{A} \tilde{x}\right\|\right. \\
& \left.+2 \varrho_{n}\left(1-\varrho_{n}\right)\left\langle-\tilde{x}, \mathbb{S} x_{n}-\tilde{x}\right\rangle+\varrho_{n}^{2}\|\tilde{x}\|^{2}\right) \\
& \leq\left[1-2\left(1-\varsigma_{n}\right) \varrho_{n}\right]\left\|x_{n}-\tilde{x}\right\|^{2} \\
& +2 \varrho_{n}\left(1-\varsigma_{n}\right) \tau_{n}\|\tilde{x}\|\left\|\mathbb{A} \mathbb{S} x_{n}-\mathbb{A} \tilde{x}\right\| \\
& +2 \varrho_{n}\left(1-\varsigma_{n}\right)\left(1-\varrho_{n}\right)\left\langle-\tilde{x}, \mathbb{S} x_{n}-\tilde{x}\right\rangle \\
& +\left(1-\varsigma_{n}\right) \varrho_{n}^{2}\left(\|\tilde{x}\|^{2}+\left\|x_{n}-\tilde{x}\right\|^{2}\right) \\
& =\left[1-2\left(1-\varsigma_{n}\right) \varrho_{n}\right]\left\|x_{n}-\tilde{x}\right\|^{2} \\
& +2\left(1-\varsigma_{n}\right) \varrho_{n}\left\{\tau_{n}\|\tilde{x}\|\left\|\mathbb{A} S x_{n}-\mathbb{A} \tilde{x}\right\|\right. \\
& +\left(1-\varrho_{n}\right)\left\langle-\tilde{x}, \mathbb{S} x_{n}-\tilde{x}\right\rangle \\
& \left.+\varrho_{n}\left(\|\tilde{x}\|^{2}+\left\|x_{n}-\tilde{x}\right\|^{2}\right)\right\} \text {. }
\end{aligned}
$$

It is clear that $\sum_{n} 2\left(1-\varsigma_{n}\right) \varrho_{n}=\infty$ and

$$
\begin{aligned}
\limsup _{n \rightarrow \infty} & \left\{\tau_{n}\|\tilde{x}\|\left\|\mathbb{A} \mathbb{S} x_{n}-\mathbb{A} \tilde{x}\right\|+\left(1-\varrho_{n}\right)\right. \\
& \left.\times\left\langle-\tilde{x}, \mathbb{S} x_{n}-\tilde{x}\right\rangle+\varrho_{n}\left(\|\tilde{x}\|^{2}+\left\|x_{n}-\tilde{x}\right\|^{2}\right)\right\} \leq 0 .
\end{aligned}
$$

By Lemma 7, we conclude that $x_{n} \rightarrow \tilde{x}$. This completes the proof.

Corollary 11. Suppose that $(\mathbb{A}+\mathbb{B})^{-1}(0) \neq \emptyset$. Let $\tau$ be a constant satisfying $a \leq \tau \leq b$, where $[a, b] \subset(0,2 \varrho)$. For $t \in(0,1-\tau /(2 \varrho))$, let $\left\{x_{t}\right\} \subset \mathscr{C}$ be a net generated by

$$
x_{t}=J_{\tau}^{\mathbb{B}}\left((1-t) x_{t}-\tau \mathbb{A} x_{t}\right) .
$$

Then the net $\left\{x_{t}\right\}$ converges strongly, as $t \rightarrow 0+$, to a point $\tilde{x}=\operatorname{proj}_{(\mathbb{A}+\mathbb{B})^{-1}(0)}(0)$ which is the minimum norm element in $(\mathbb{A}+\mathbb{B})^{-1}(0)$.

Corollary 12. Suppose that $(\mathbb{A}+\mathbb{B})^{-1}(0) \neq \emptyset$. For given $x_{0} \in$ $\mathscr{C}$, let $\left\{x_{n}\right\} \subset \mathbb{C}$ be a sequence generated by

$$
x_{n+1}=\varsigma_{n} x_{n}+\left(1-\varsigma_{n}\right) J_{\tau_{n}}^{\mathbb{B}}\left(\left(1-\varrho_{n}\right) x_{n}-\tau_{n} \mathbb{A} x_{n}\right)
$$

for all $n \geq 0$, where $\left\{\tau_{n}\right\} \subset(0,2 \varrho),\left\{\varrho_{n}\right\} \subset(0,1)$, and $\left\{\varsigma_{n}\right\} \subset$ $(0,1)$ satisfy

(i) $\lim _{n \rightarrow \infty} \varrho_{n}=0$ and $\sum_{n} \varrho_{n}=\infty$;

(ii) $0<\underline{\lim }_{n \rightarrow \infty} \varsigma_{n} \leq \overline{\lim }_{n \rightarrow \infty} \varsigma_{n}<1$;

(iii) $a\left(1-\varrho_{n}\right) \leq \tau_{n} \leq b\left(1-\varrho_{n}\right)$, where $[a, b] \subset(0,2 \varrho)$ and $\lim _{n \rightarrow \infty}\left(\tau_{n+1}-\tau_{n}\right)=0$.

Then $\left\{x_{n}\right\}$ converges strongly to a point $\tilde{x}=\operatorname{proj}_{(\mathbb{A}+\mathbb{B})^{-1}(0)}(0)$ which is the minimum norm element in $(\mathbb{A}+\mathbb{B})^{-1}(0)$.

\section{Conflict of Interests}

The authors declare that there is no conflict of interests regarding the publication of this paper.

\section{Acknowledgments}

The authors would like to thank the editor and the referees for the useful comments and suggestions. Sun Young Cho was supported by the Basic Science Research Program through the National Research Foundation of Korea funded by the Ministry of Education, Science and Technology (KRF2013053358). Li-Jun Zhu was supported in part by NNSF of China (61362033 and NZ13087).

\section{References}

[1] S. M. Robinson, "Generalized equations and their solutions. I. Basic theory," Mathematical Programming Study, no. 10, pp. 128141, 1979.

[2] R. T. Rockafellar, "Monotone operators and the proximal point algorithm," SIAM Journal on Control and Optimization, vol. 14, no. 5, pp. 877-898, 1976.

[3] H. H. Bauschke and P. L. Combettes, "A Dykstra-like algorithm for two monotone operators," Pacific Journal of Optimization, vol. 4, no. 3, pp. 383-391, 2008.

[4] P. L. Combettes and S. A. Hirstoaga, "Approximating curves for nonexpansive and monotone operators," Journal of Convex Analysis, vol. 13, no. 3-4, pp. 633-646, 2006. 
[5] P. L. Combettes and S. A. Hirstoaga, "Visco-penalization of the sum of two monotone operators," Nonlinear Analysis, vol. 69, no. 2, pp. 579-591, 2008.

[6] J. Eckstein and D. P. Bertsekas, "On the Douglas-Rachford splitting method and the proximal point algorithm for maximal monotone operators," Mathematical Programming, vol. 55, no. 3, pp. 293-318, 1992.

[7] P. Lions and B. Mercier, "Splitting algorithms for the sum of two nonlinear operators," SIAM Journal on Numerical Analysis, vol. 16, no. 6, pp. 964-979, 1979.

[8] A. Moudafi, "On the regularization of the sum of two maximal monotone operators," Nonlinear Analysis: Theory, Methods \& Applications A, vol. 42, pp. 1203-1208, 2000.

[9] N. Onjai-uea and P. Kumam, "A new iterative scheme for equilibrium problems, fixed point problems for nonexpansive mappings and maximal monotone operators," Fixed Point Theory and Applications, vol. 2012, article 27, 2012.

[10] G. B. Passty, "Ergodic convergence to a zero of the sum of monotone operators in Hilbert space," Journal of Mathematical Analysis and Applications, vol. 72, no. 2, pp. 383-390, 1979.

[11] W. Phuengrattana, S. Suantai, K. Wattanawitoon, U. Witthayarat, and P. Kumam, "Weak and strong convergence theorems of proximal point algorithm for solving generalized mixed equilibrium problems and finding zeroes of maximal monotone operators in Banach spaces," Journal of Computational Analysis and Applications, vol. 16, no. 2, pp. 264-281, 2014.

[12] S. Saewan, P. Kumam, and Y. J. Cho, "Strong convergence for maximal monotone operators, relatively quasi-nonexpansive mappings, variational inequalities and equilibrium problems," Journal of Global Optimization, vol. 57, no. 4, pp. 1299-1318, 2013.

[13] S. Saewan, P. Kumam, and Y. J. Cho, "Convergence theorems for finding zero points of maximal monotone operators and equilibrium problems in Banach spaces," Journal of Inequalities and Applications, vol. 2013, article 247, 18 pages, 2013.

[14] S. Saewan, P. Kumam, and P. Kanjanasamranwong, "The hybrid projection algorithm for finding the common fixed points of nonexpansive mappings and the zeroes of maximal monotone operators in Banach spaces," Optimization, vol. 63, no. 9, pp. 1319-1338, 2014.

[15] P. Sunthrayuth and P. Kumam, "A system of generalized mixed equilibrium problems, maximal monotone operators, and fixed point problems with application to optimization problems," Abstract and Applied Analysis, vol. 2012, Article ID 316276, 39 pages, 2012.

[16] K. Wattanawitoon and P. Kumam, "Hybrid proximal-point methods for zeros of maximal monotone operators, variational inequalities and mixed equilibrium problems," International Journal of Mathematics and Mathematical Sciences, vol. 2011, Article ID 174796, 31 pages, 2011.

[17] K. Wattanawitoon and P. Kumam, "Generalized mixed equilibrium problems for maximal monotone operators and two relatively quasi-nonexpansive mappings," Thai Journal of Mathematics, vol. 9, no. 1, pp. 165-189, 2011.

[18] K. Wattanawitoon and P. Kumam, "A new iterative scheme for generalized mixed equilibrium, variational inequality problems, and a zero point of maximal monotone operators," Journal of Applied Mathematics, vol. 2012, Article ID 692829, 27 pages, 2012.

[19] K. Wattanawitoon and P. Kumam, "Modified proximal point algorithms for finding a zero point of maximal monotone operators, generalized mixed equilibrium problems and variational inequalities,' Journal of Inequalities and Applications, vol. 2012, article 118, 2012.

[20] U. Witthayarat, Y. J. Cho, and P. Kumam, "Convergence of an iterative algorithm for common solutions for zeros of maximal accretive operator with applications," Journal of Applied Mathematics, vol. 2012, Article ID 185104, 17 pages, 2012.

[21] S. Zhang, J. H. W. Lee, and C. K. Chan, "Algorithms of common solutions to quasi variational inclusion and fixed point problems," Applied Mathematics and Mechanics. English Edition, vol. 29, no. 5, pp. 571-581, 2008.

[22] S. Takahashi, W. Takahashi, and M. Toyoda, "Strong convergence theorems for maximal monotone operators with nonlinear mappings in Hilbert spaces," Journal of Optimization Theory and Applications, vol. 147, no. 1, pp. 27-41, 2010.

[23] K. Goebel and W. A. Kirk, Topics in Metric Fixed Point Theory, vol. 28 of Cambridge Studies in Advanced Mathematics, Cambridge University Press, Cambridge, UK, 1990.

[24] T. Suzuki, "Strong convergence theorems for infinite families of nonexpansive mappings in general Banach spaces," Fixed Point Theory and Applications, vol. 2005, no. 1, pp. 103-123, 2005.

[25] H. K. Xu, "Iterative algorithms for nonlinear operators," Journal of the London Mathematical Society, vol. 66, no. 1, pp. 240-256, 2002. 


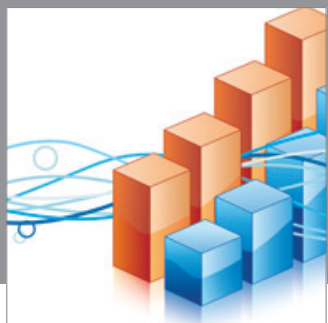

Advances in

Operations Research

mansans

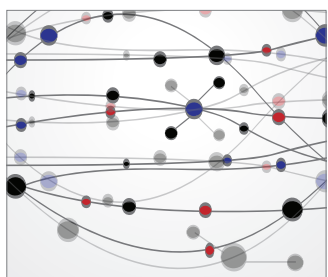

The Scientific World Journal
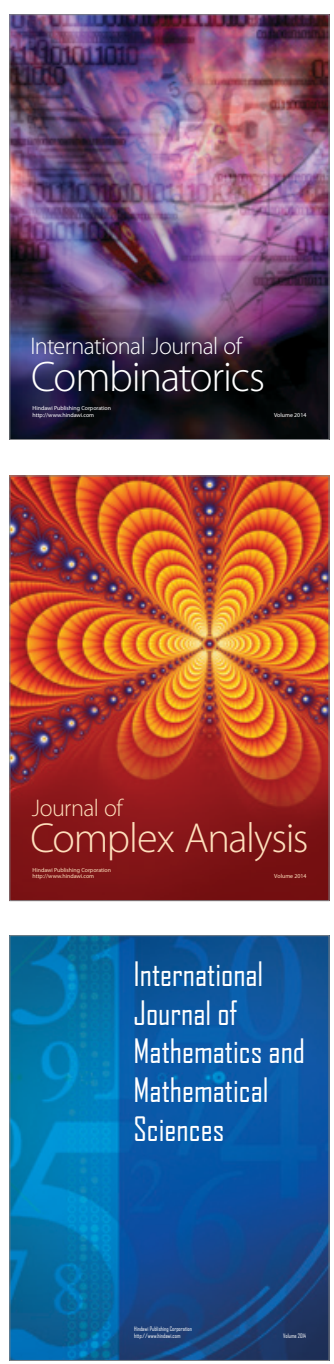
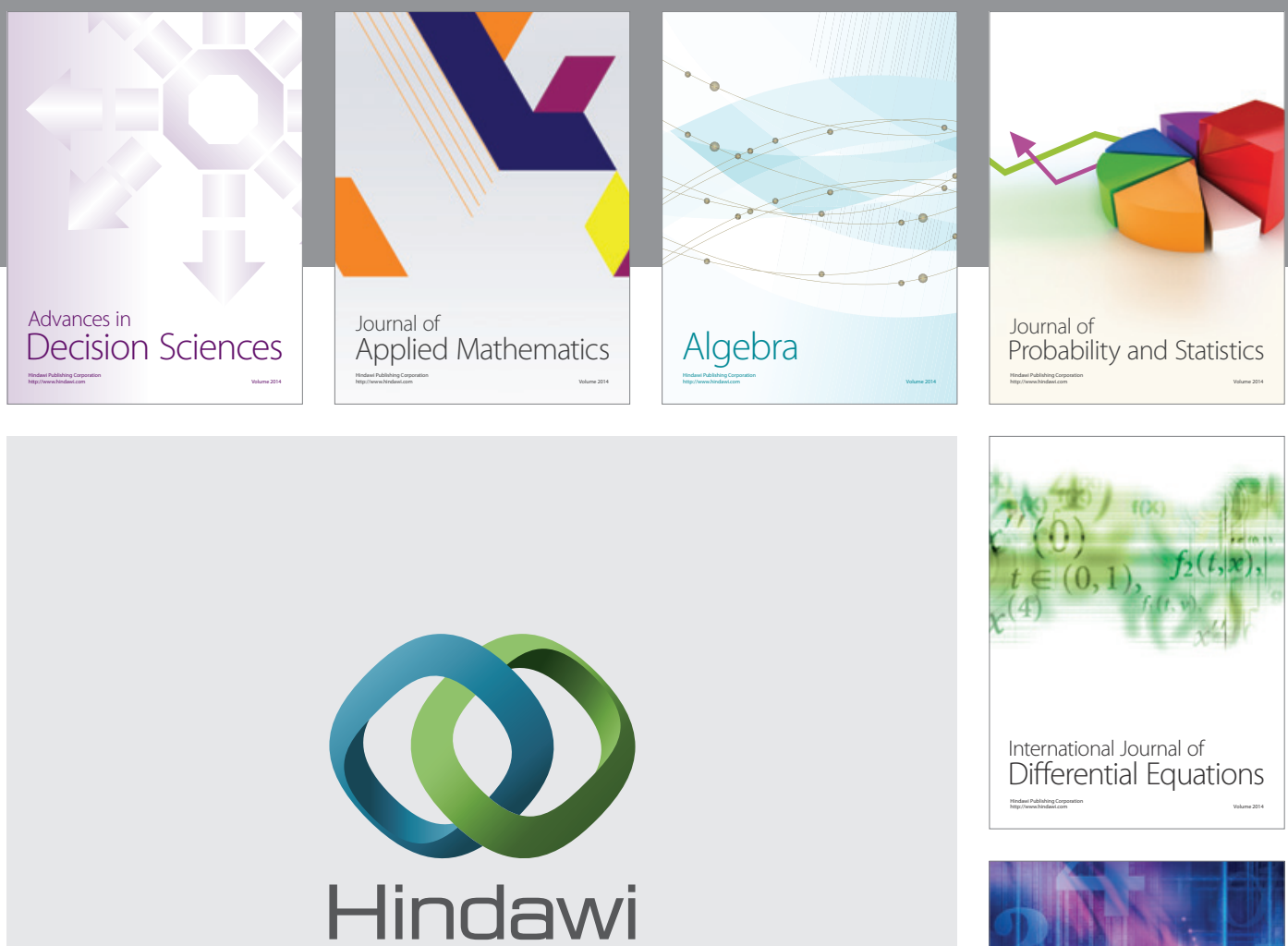

Submit your manuscripts at http://www.hindawi.com
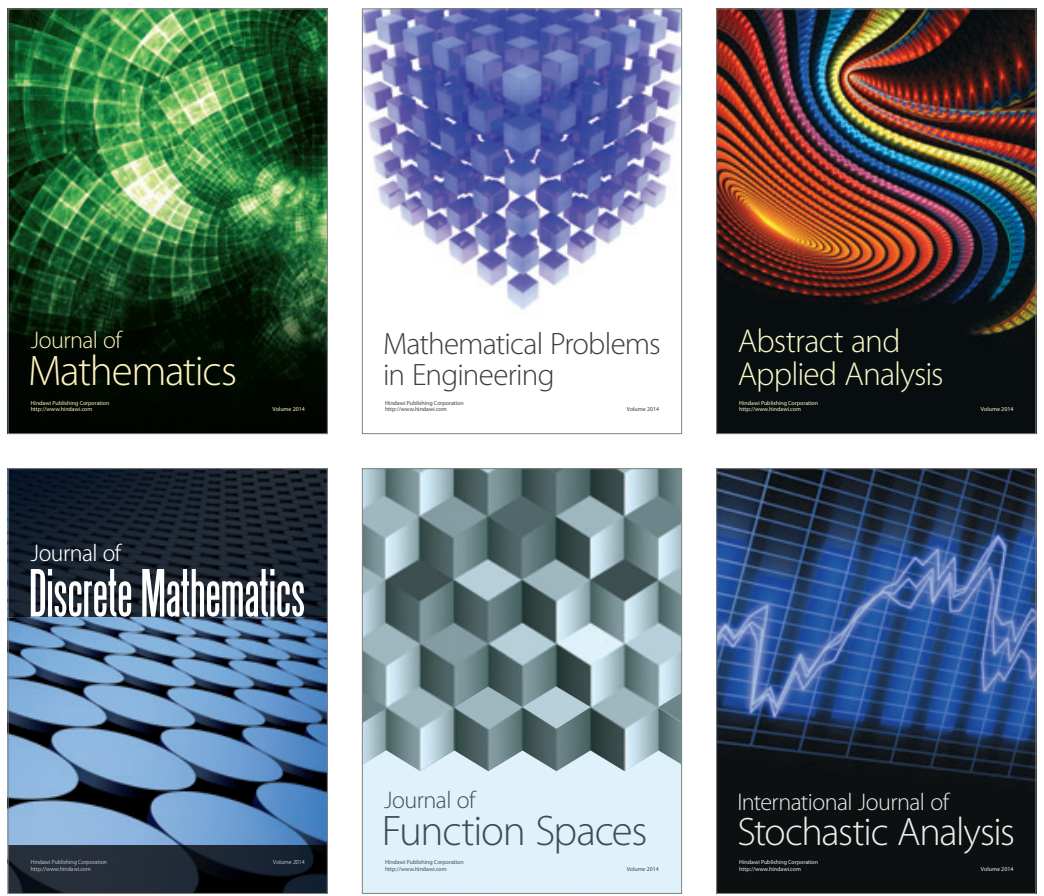

Journal of

Function Spaces

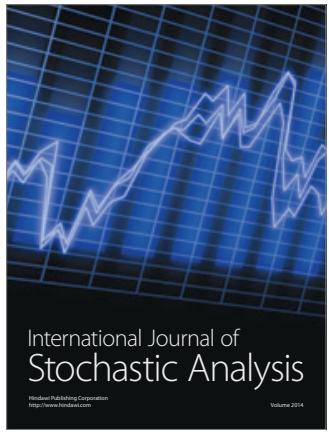

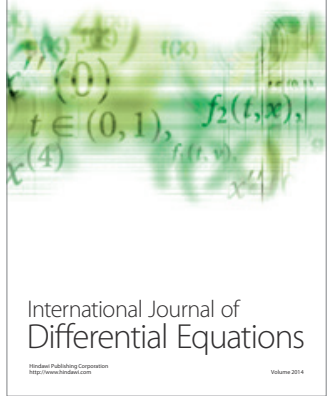
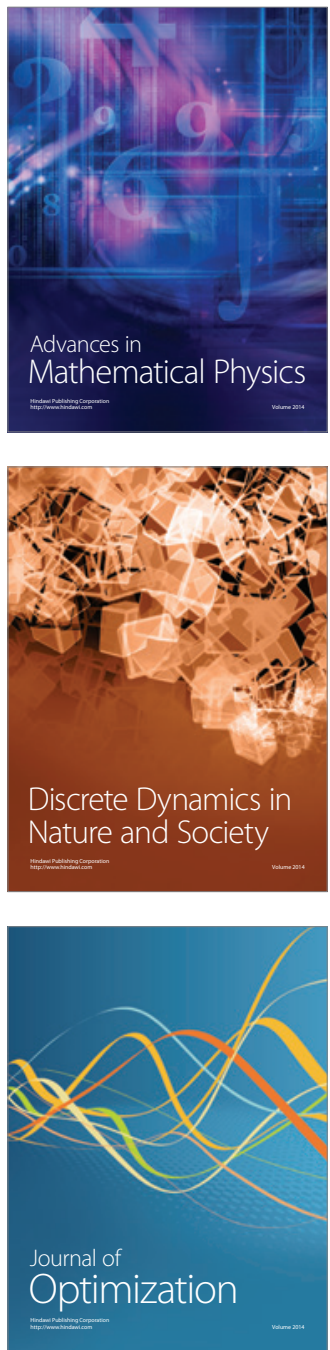